\title{
Simultaneous Detection and Estimation of Catechol, Hydroquinone, and Resorcinol in Binary and Ternary Mixtures Using Electrochemical Techniques
}

\author{
Md. Uzzal Hossain, Md. Toufiqur Rahman, and Md. Qamrul Ehsan \\ Department of Chemistry, University of Dhaka, Dhaka 1000, Bangladesh \\ Correspondence should be addressed to Md. Qamrul Ehsan; mqehsan@du.ac.bd
}

Received 10 September 2015; Accepted 22 November 2015

Academic Editor: Adil Denizli

Copyright ( $) 2015$ Md. Uzzal Hossain et al. This is an open access article distributed under the Creative Commons Attribution License, which permits unrestricted use, distribution, and reproduction in any medium, provided the original work is properly cited.

Cyclic voltammetry (CV) and differential pulse voltammetry (DPV) were performed with a glassy carbon electrode (GCE) modified with polyglutamic acid (PGA) on the three dihydroxybenzene isomers, catechol (CT), hydroquinone (HQ), and resorcinol (RS). At bare GCE, these isomers exhibited voltammograms with highly overlapped redox peaks that impeded their simultaneous detection in binary and ternary mixtures. On the contrary, at PGA modified GCE binary and ternary mixtures of the dihydroxybenzene isomers showed well-resolved redox peaks in both CV and DPV experiments. This resolving ability of PGA modified GCE proves its potential to be exploited as an electrochemical sensor for the simultaneous detection of these isomers.

\section{Introduction}

The isomers of dihydroxybenzene catechol (1,2-dihydroxybenzene, CT), hydroquinone (1,4-dihydroxybenzene, HQ), and resorcinol (1,3-dihydroxybenzene, RS) are widely used in medicines, pesticides, cosmetics, tanning, flavoring agents, antioxidants, dyes, and photography chemicals $[1,2]$. Due to their high toxicity and low degradability in the ecological environment, they are considered as environmental pollutants $[3,4]$. Moreover, these isomers are often coexisting in environmental samples $[5,6]$ and interfere with each other during their identification [7]. Therefore, it is necessary to develop simple and rapid analytical method for the detection and determination of these isomers in a mixture.

Many analytical methods have been established to determine dihydroxybenzene isomers, such as HPLC [8], fluorescence [9], chemiluminescence [10], spectrophotometry [11], GC-MS [12], and electrochromatography [13]. Among them, electrochemical methods have attracted ever-growing attention due to the advantages such as fast response, low cost, simple operation, faster analysis, high sensitivity, and excellent selectivity [14]. At ordinary bare electrodes, these isomers show highly overlapping voltammograms where their redox peaks are not resolved [15-20]. Detection and estimations of $\mathrm{CT}$ and $\mathrm{HQ}$ in a binary mixture have been reported in literature [21-28]. However, the simultaneous determination of CT, RS, and HQ in a ternary mixture by electrochemical methods is insufficient. Recently, though the simultaneous determinations of $\mathrm{CT}, \mathrm{HQ}$, and RS have been performed by single-wall carbon nanotube (SWCNT) [29], modified glassy carbon electrode (GCE), modified multielectrode array [30], and graphite doped carbon ionic liquid electrode [25], it is still worthwhile to investigate novel electrode material for the simultaneous determination of CT, HQ, and RS in ternary mixture. In this paper, an effective and practical method for the simultaneous detections and quantitative estimation of CT, HQ, and RS using CV and DPV techniques at PGA modified GCE is presented.

\section{Experimental Section}

2.1. Reagents and Apparatus. Chemicals used in this study are (i) catechol (BDH); (ii) hydroquinone $(\mathrm{BDH})$; (iii) resorcinol (BDH); (iv) $\mathrm{NaOH}$ pellets (Merck, Germany); (v) potassium chloride (Merck, Germany); (vi) potassium phosphate (monobasic) (Merck, Germany); (vii) L-glutamic acid 
(viii); sodium acetate (Merck, Germany); and (ix) acetic acid (Sigma-Aldrich). Deionized water was used for solution preparation and cleaning purposes. Solutions were purged with $99.997 \%$ dry nitrogen (BOC, Bangladesh) to remove dissolved oxygen and to maintain inert atmosphere prior to experiment. All reagents were obtained as AR grade and used without further purification.

This study was carried out using an Epsilon Electroanalyser developed by Bioanalytical System, Inc., USA, in a Pyrex glass microcell with Teflon cap. A glassy carbon electrode (GCE) was used as working electrode while $\mathrm{Ag} / \mathrm{AgCl}$ and Pt wire functioned as the reference and counter electrodes, respectively. The $\mathrm{pH}$ of solutions was measured using Orion 2 Star (made by Thermo Electron Corporation) $\mathrm{pH}$ meter.

2.2. Electrode Modification. The bare glassy carbon electrode was first polished on polishing cloth with $0.3 \mu \mathrm{m}$ alumina and then washed with distilled water and sonicated in ethanol. The cleaned and polished GCE was placed in $0.01 \mathrm{M}$ glutamic acid solution in a $\mathrm{pH} 7.0$ phosphate buffer, which was previously purged with high purity nitrogen for 10 minutes. The electrode was treated with four cycles of $\mathrm{CV}$ between -1.5 and $2.0 \mathrm{~V}$ at a scan rate of $100 \mathrm{mV} / \mathrm{s}$. A uniform adherent blue polymeric layer was observed on the electrode surface. The electrode was ready for the experiment after a rinse with deionized water. Voltammograms correspond to $1.0 \mathrm{mmol} \cdot \mathrm{L}^{-1}$ of analyte at $50 \mathrm{mV} \cdot \mathrm{s}^{-1}$ scan rate unless otherwise mentioned.

\section{Results and Discussion}

3.1. Behavior of Dihydroxybenzenes at Bare and Modified GCE. At bare GCE in acetate buffer solution (ABS, pH 4.5) CT and HQ showed highly overlapping character where their anodic and cathodic peaks merged forming an overall voltammogram that impeded the simultaneous detection using CV at bare GCE in a binary mixture (Figure 1(a)). We observed similar behavior in UV-Vis spectroscopy. CT and HQ showed maximum absorbance at $275.45 \mathrm{~nm}$ and $288.60 \mathrm{~nm}$ wavelengths, respectively, in ABS. But in their binary mixture only one peak was observed at $278.20 \mathrm{~nm}$ which is the combined absorbance of catechol and hydroquinone. On the other hand at PGA modified GCE, both CT and HQ showed highly reversible behavior (ipa/ipc close to unity) in $\mathrm{CV}$ with dramatic signal enhancement compared to that at bare GCE (Figures 1(b) and 1(c)).

Figure $1(\mathrm{~d})$ shows the $\mathrm{CV}$ of CT-HQ binary mixture at PGA-GCE overlaid with that of individual CT and HQ. It is clear that both $\mathrm{CT}$ and HQ retained their corresponding redox peaks in the mixture. A comparison between the CV responses of the binary mixture of $\mathrm{CT}$ and $\mathrm{HQ}$ at bare and PGA modified GCE is depicted in Figure 1(e). Similar behavior was observed in phosphate buffer solution (PBS, $\mathrm{pH}$ 7.0).

3.2. Electrochemical Behavior of Dihydroxybenzenes in Binary Mixtures at the Modified GCE. A series of CV and DPV experiments were performed on binary mixtures of CT, HQ, and RS in PBS pH 7.0 for their simultaneous detection in the presence of another.

3.2.1. Detection of CT and HQ in Mixture in PBS. At PGA modified GCE, CT and HQ showed well separated anodic peaks at $+252 \mathrm{mV}$ and $+364 \mathrm{mV}$ and two cathodic peaks at $+264 \mathrm{mV}$ and $+151 \mathrm{mV}$, respectively. An overlay of CVs of individual CT and HQ with that of CT-HQ binary mixture (Figure 2(a)) shows that both CT and HQ retain their corresponding redox peaks in the binary mixture at the modified GCE.

A DPV of the CT-HQ binary mixture in PBS is shown in Figure 2(b), which clearly shows that CT and HQ response with well separated anodic peaks while retaining their individual peak positions (at $+296 \mathrm{mV}$ and $+193 \mathrm{mV}$, resp.) at the modified GCE. The peak separation is measured to be $103 \mathrm{mV}$ that is quite good for the simultaneous detection of these isomers in the presence of another.

3.2.2. Detection of CT and RS in Mixture in PBS. Similar experiments were done on CT-RS binary mixture. In CV, CT and RS gave responses with large peak separation that was retained in the binary mixture (Figure 3(a)).

In DPV, CT and RS showed oxidation peaks (at $+293 \mathrm{mV}$ and $+702 \mathrm{mV}$ ) separated by $409 \mathrm{mV}$ that is well suited for their simultaneous detection at PGA modified GCE (Figure 2(b)).

3.2.3. Detection of HQ and RS in Mixture in PBS. Same experiments were performed on HQ-RS binary mixture that exhibited even better peak separation in both CV and DPV depicted in Figures 4(a) and 4(b), respectively. In DPV, the binary mixture shows two anodic peaks at $+202 \mathrm{mV}$ and $+701 \mathrm{mV}$, respectively, resulting in peak separation of $499 \mathrm{mV}$.

3.3. Detection of CT, HQ, and RS in Ternary Mixture. CV and DPV experiments were performed to detect CT, HQ, and RS simultaneously from ternary mixtures in both acetate buffer (ABS) and phosphate buffer solution (PBS) at PGA modified GCE.

3.3.1. Detection of CT, HQ, and RS in Ternary Mixture in PBS. Cyclic voltammograms of individual CT, HQ, and RS and that of the mixture in PBS at PGA-GCE are overlaid on Figure 5(a). Three anodic peaks at $+94 \mathrm{mV},+201 \mathrm{mV}$, and $+605 \mathrm{mV}$ and two cathodic peaks at $+134 \mathrm{mV}$ and $+19 \mathrm{mV}$ were observed. These peaks correspond to the anodic and cathodic peaks of CT, HQ, and RS.

The DPV study of CT, HQ, and RS as individual isomers and as a mixture is shown in Figure 5(b), which shows that at PGA modified GCE, CT, HQ, and RS retained their corresponding anodic peaks at $+295 \mathrm{mV},+202 \mathrm{mV}$, and $+715 \mathrm{mV}$, respectively, in ternary mixture.

3.4. Detection of CT, HQ, and RS in Ternary Mixture in ABS. The CVs of the ternary mixture and individual CT, HQ, and RS in acetate buffer solution at PGA modified GCE were taken and are shown in Figure 6(a). Three anodic peaks at $+247 \mathrm{mV},+354 \mathrm{mV}$, and $+769 \mathrm{mV}$ for the three isomers and 


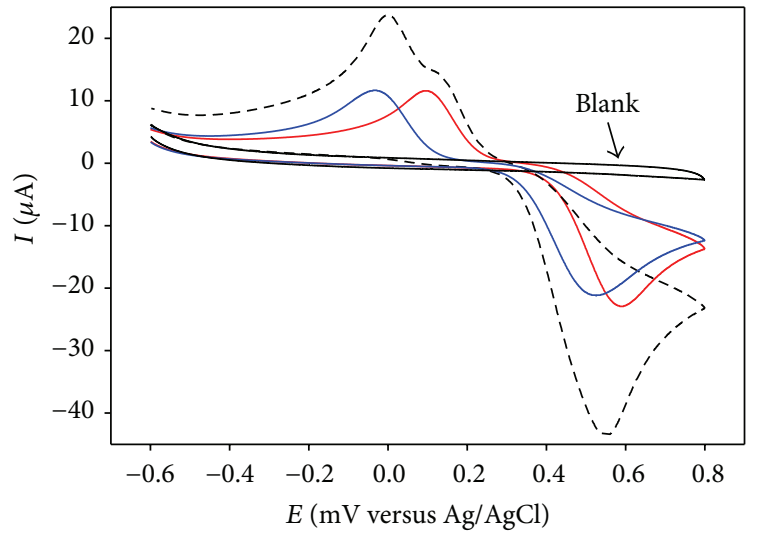

- CT at bare GCE - - - CT + HQ at bare GCE - HQ at bare GCE

(a)

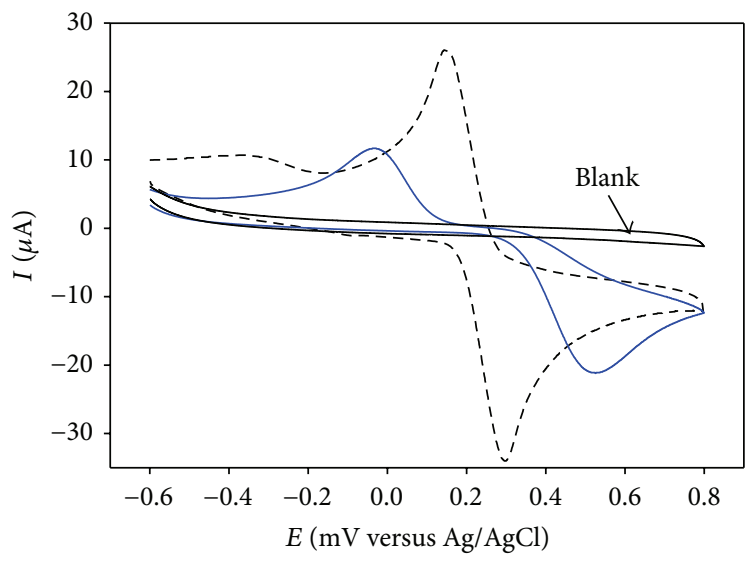

- HQ at bare GCE

-- HQ at PGA-GCE

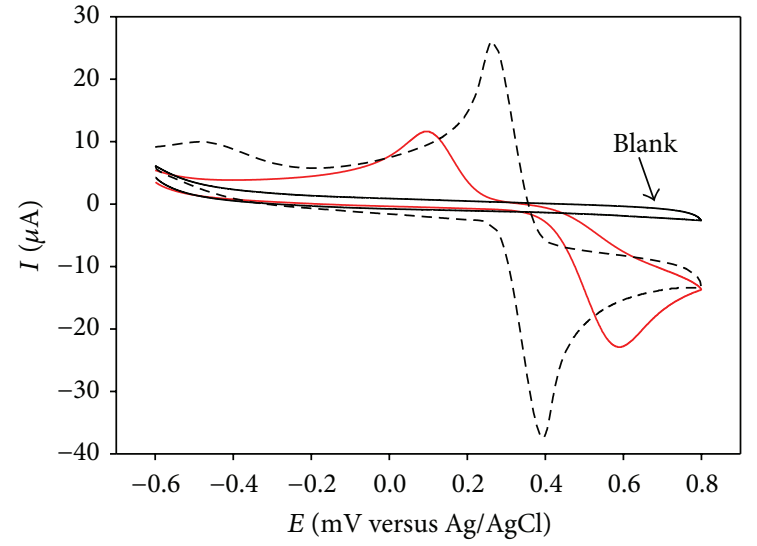

- CT at bare GCE

- - CT at PGA-GCE

(b)

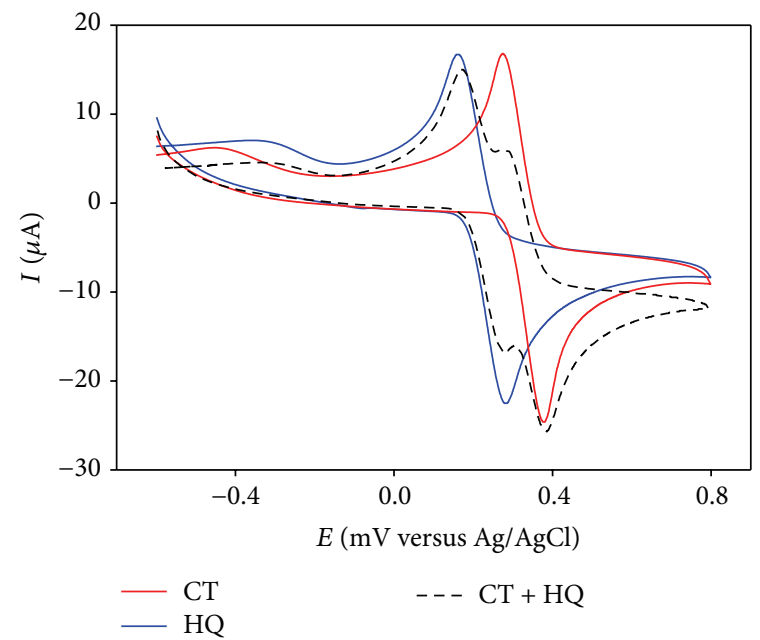

(d)

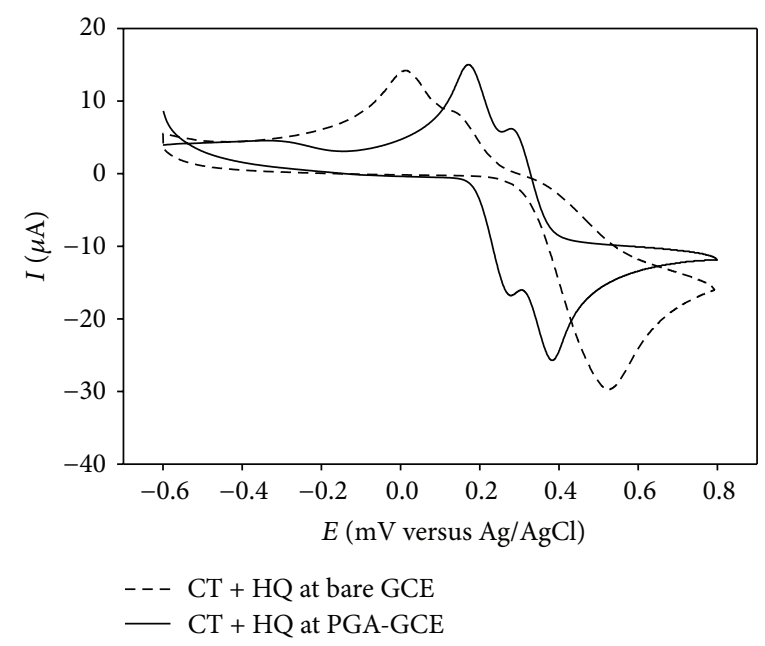

(e)

Figure 1: CVs of (a) CT and HQ (solid lines), CT-HQ binary mixture (dashed line) at bare GCE; (b) CT at bare GCE (solid line) and at PGA-GCE (dashed line); (c) HQ at bare GCE (solid line) and at PGA-GCE (dashed line); (d) overlay of individual CT and HQ (solid lines) and CT-HQ binary mixture (dashed line) at PGA-GCE; (e) comparison between simultaneous CT and HQ at bare GCE (dashed) and at PGA-GCE (solid line) in ABS pH 4.5. 


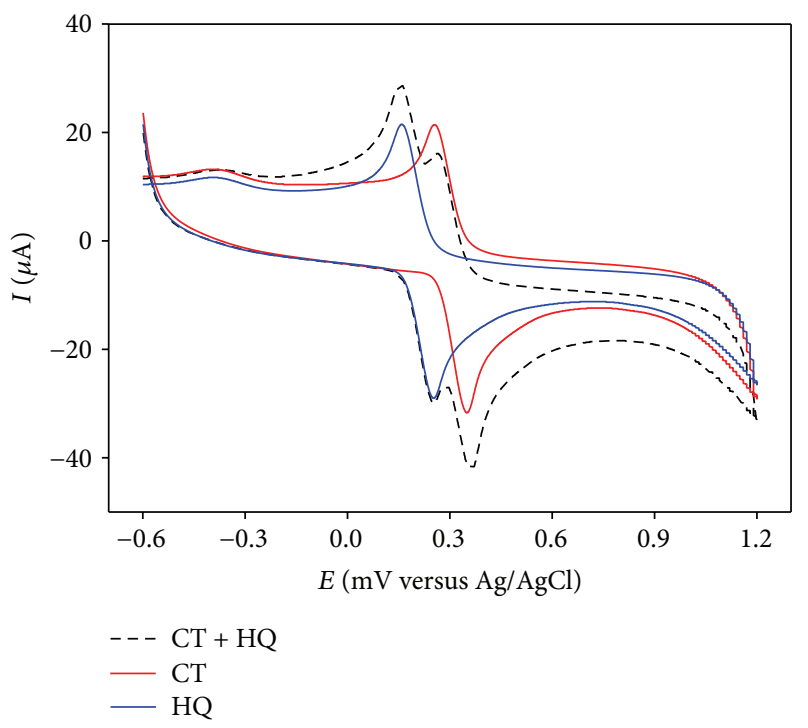

(a)

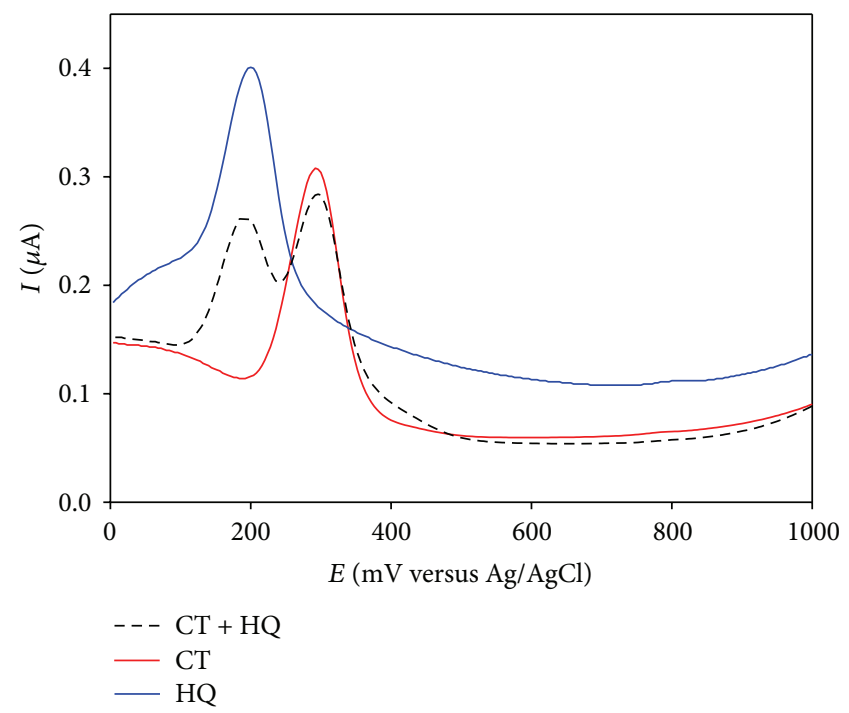

(b)

FIGURE 2: Overlay of (a) CV and (b) DPV responses of individual (solid lines) and binary mixture (dashed line) of CT and HQ in PBS at PGA modified GCE.

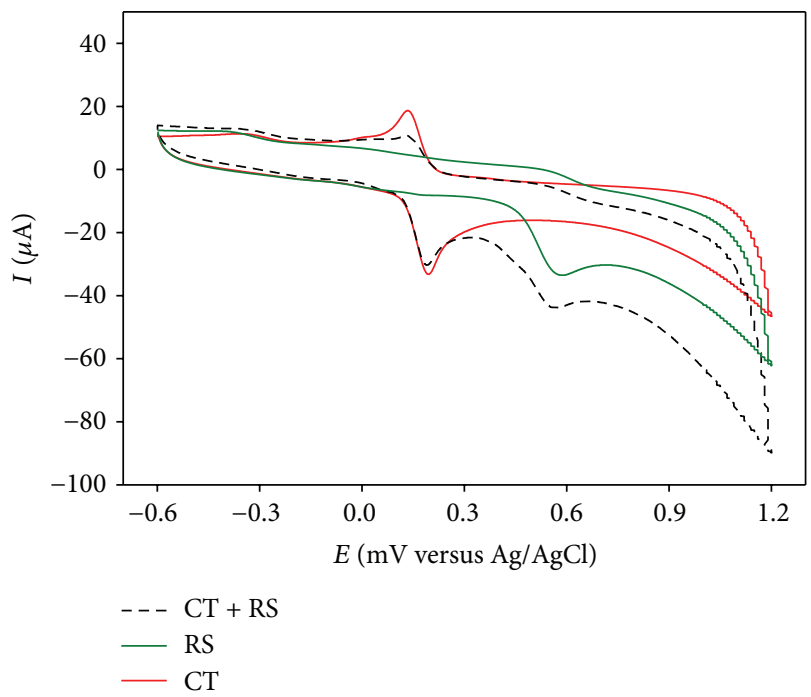

(a)

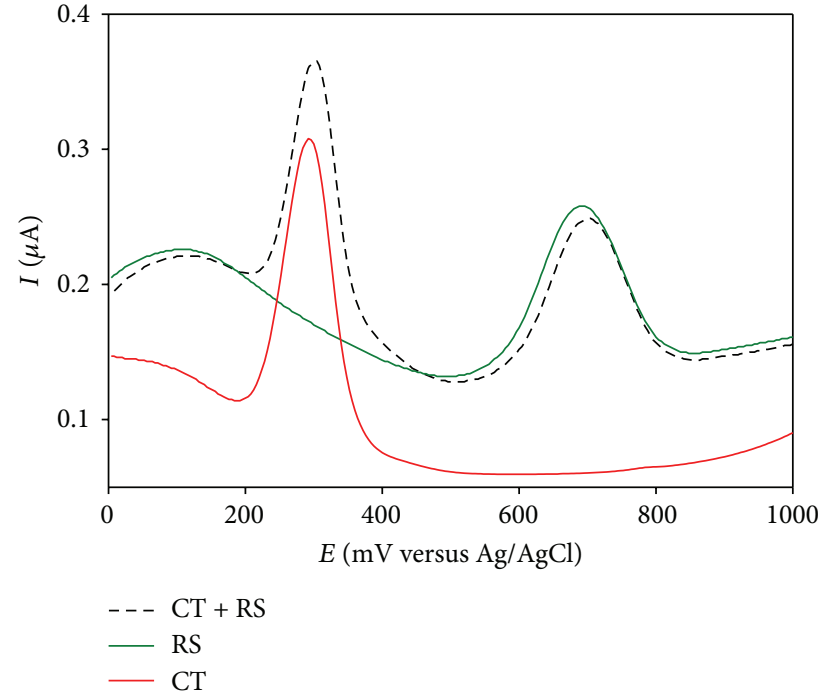

(b)

FIGURE 3: Overlay of (a) CV and (b) DPV responses of individual (solid lines) and mixture (dashed line) of CT and RS in PBS at PGA modified GCE.

two cathodic peaks at $+224 \mathrm{mV}$ and $+135 \mathrm{mV}$ for CT and HQ, respectively, were found.

Figure 6(b) shows the overlay of DPV responses of individual CT, HQ, and $\mathrm{RS}$ (at $+138 \mathrm{mV},+34 \mathrm{mV}$, and $+513 \mathrm{mV}$ resp.) and that of ternary mixture in ABS.

\subsection{Quantitative Estimation}

3.5.1. Quantitative Estimation of CT in the Presence of HQ. DPV was performed on the binary mixture of catechol and hydroquinone at PGA modified GCE within the potential range of $+100 \mathrm{mV}$ to $+400 \mathrm{mV}$. $10.0 \mathrm{~mL}$ of analyte solutions was prepared to vary the amount of $1.0 \mathrm{mmol} \cdot \mathrm{L}^{-1}$ solution CT with successive increment of $10.0 \mu \mathrm{L}$ using a micropipette while keeping HQ concentrations constant at $1.0 \mathrm{mmol} \cdot \mathrm{L}^{-1}$. The resulting DPVs are shown in Figure 7(a).

A calibration curve (Figure 7(b)) was drawn for different concentrations of catechol. This calibration curve can be used to quantify catechol (CT) in the presence of hydroquinone (HQ) in a binary mixture. The detection limit of catechol in presence of hydroquinone was found in micromolar range.

3.5.2. Quantitative Estimation of HQ in the Presence of CT and RS. DPV was performed on a ternary mixture of CT and 


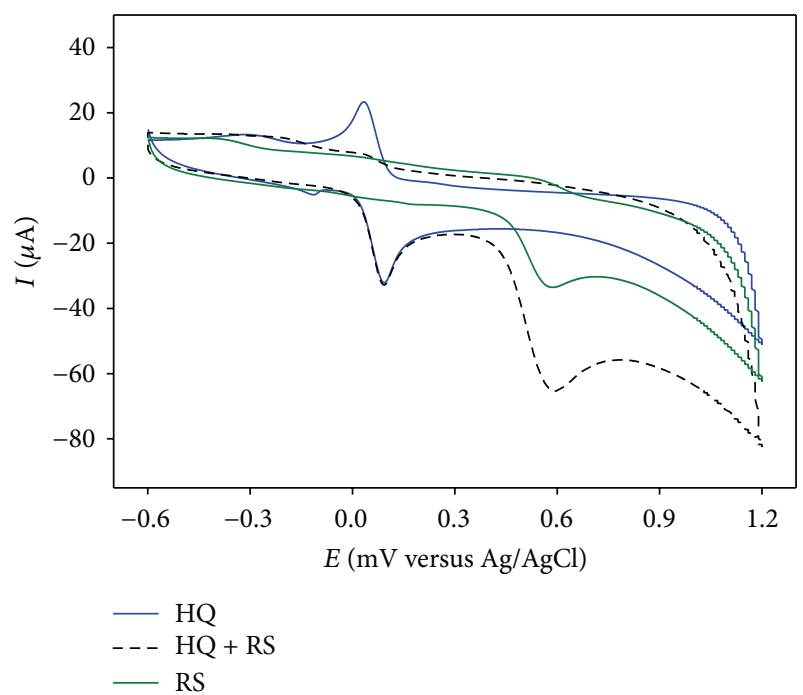

(a)

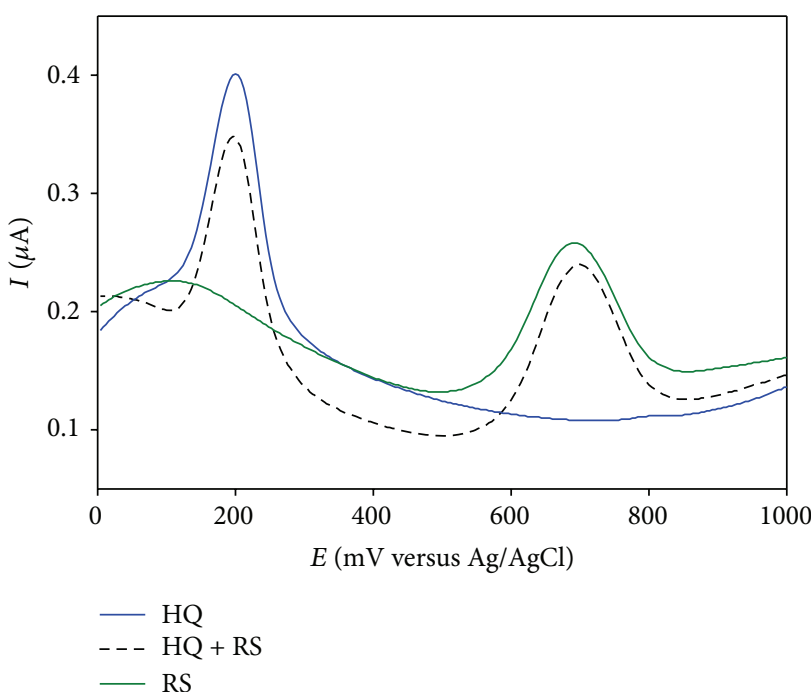

(b)

FIgure 4: Overlay of (a) CV and (b) DPV responses of individual (solid lines) and mixture (dashed line) of HQ and RS in PBS at PGA modified GCE.

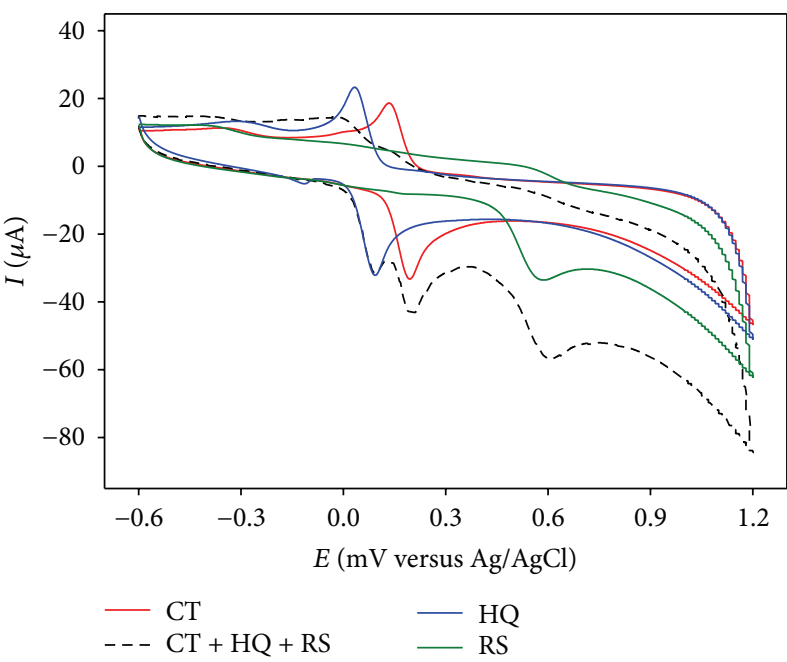

(a)

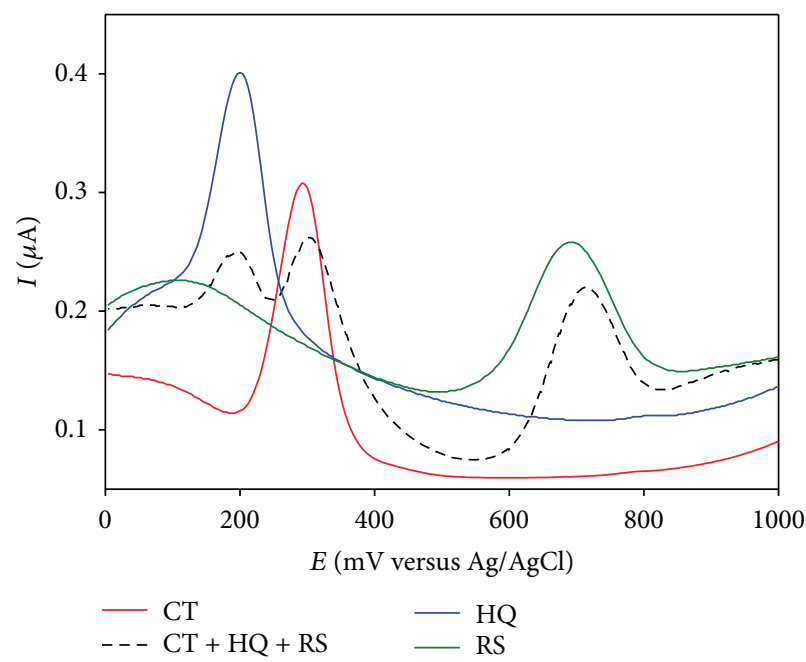

(b)

FIgURE 5: Overlay of (a) CV and (b) DPV responses of individual (solid lines) and ternary mixture (dashed line) of CT, HQ, and RS in PBS at PGA modified GCE.

HQ and RS at PGA modified GCE in the potential range of $-200 \mathrm{mV}$ to $+800 \mathrm{mV}$. $10.0 \mathrm{~mL}$ of analyte solutions was prepared to vary the amount of $1.0 \mathrm{mmol} \cdot \mathrm{L}^{-1}$ solution HQ with successive increment of $10.0 \mu \mathrm{L}$ using a micropipette while keeping CT and RS concentrations constant at $1.0 \mathrm{mmol} \cdot \mathrm{L}^{-1}$. The resulting DPVs are shown in Figure 8(a). A calibration curve (Figure 8(b)) was drawn for different concentrations of HQ which can be used for its quantitative estimation in the presence of CT and RS. The detection limit of HQ in the presence of other isomers was found in micromolar range.

This separating ability of the polyglutamic acid modified glassy carbon electrode can be used to estimate catechol, hydroquinone, and resorcinol quantitatively in presence of others.

\section{Conclusion}

In summary, a simple yet effective method for the simultaneous detection and estimation of catechol, hydroquinone, and resorcinol using electrochemical techniques has been presented. Catechol, hydroquinone, and resorcinol have been detected from binary and ternary mixtures in ABS and PBS at PGA modified GCE. This method could be used to detect 


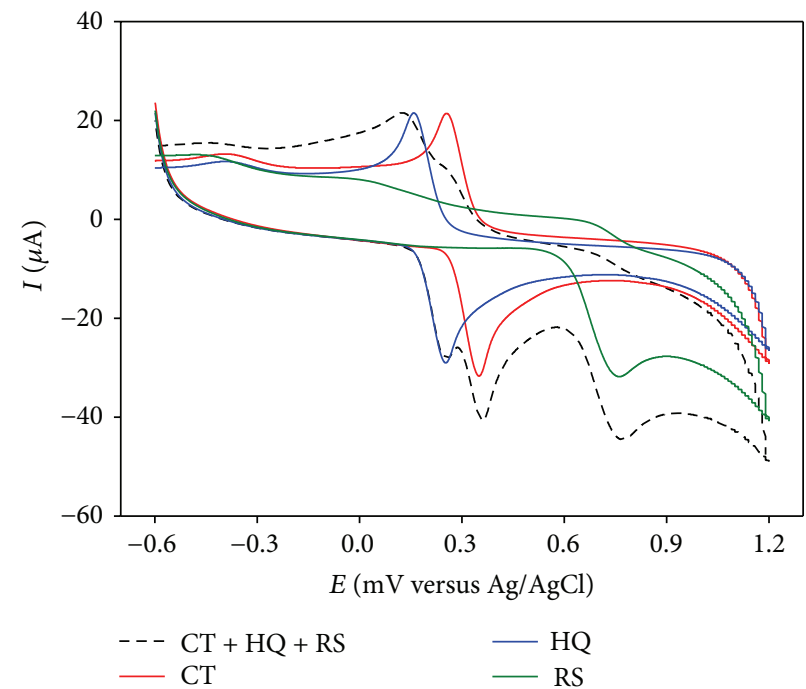

(a)

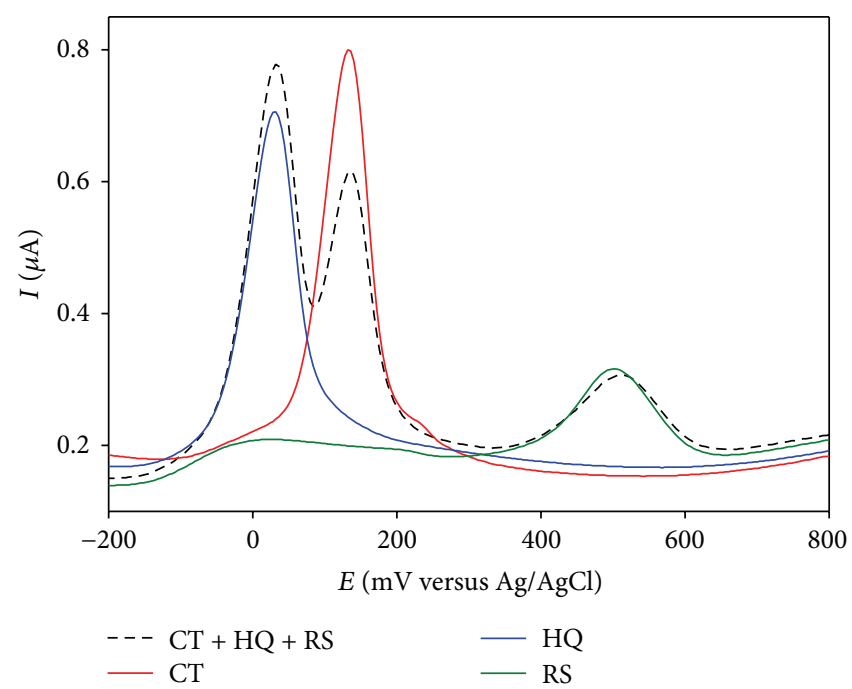

(b)

Figure 6: (a) CVs of individual (solid lines) and ternary mixture (dashed line) of CT, HQ, and RS; (b) DPV responses of individual (solid lines) and ternary mixture (dashed line) of CT, HQ, and RS in ABS at PGA modified GCE.

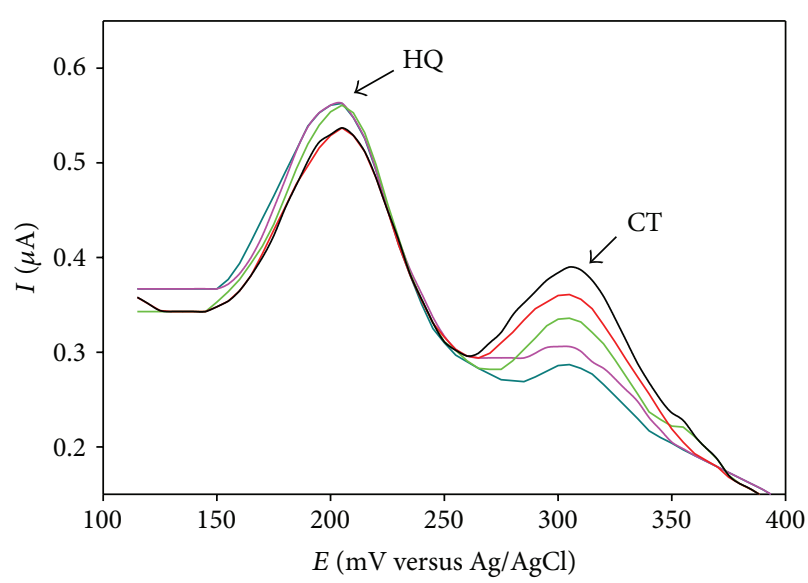

(a)

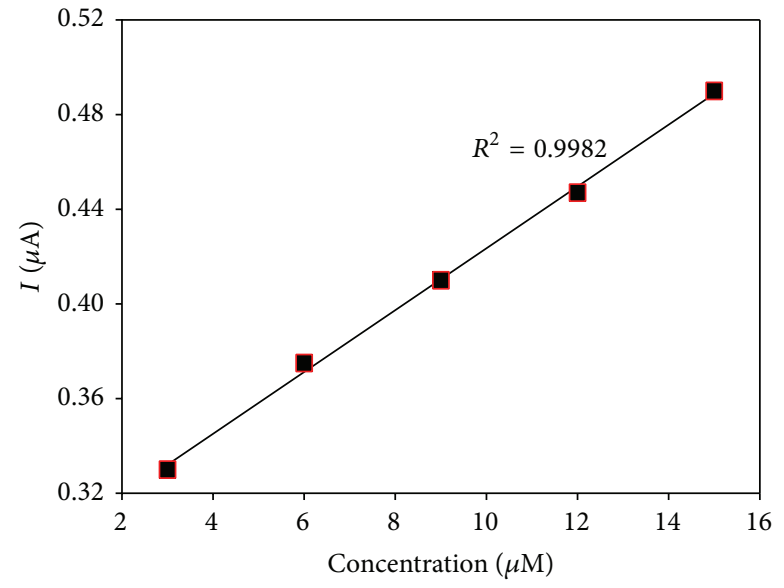

(b)

FIGURE 7: (a) Differential pulse voltammogram (DPV) of CT in the presence of HQ at PGA modified GCE in PBS. (b) Calibration curve for the quantitative determination of CT in the presence of HQ.

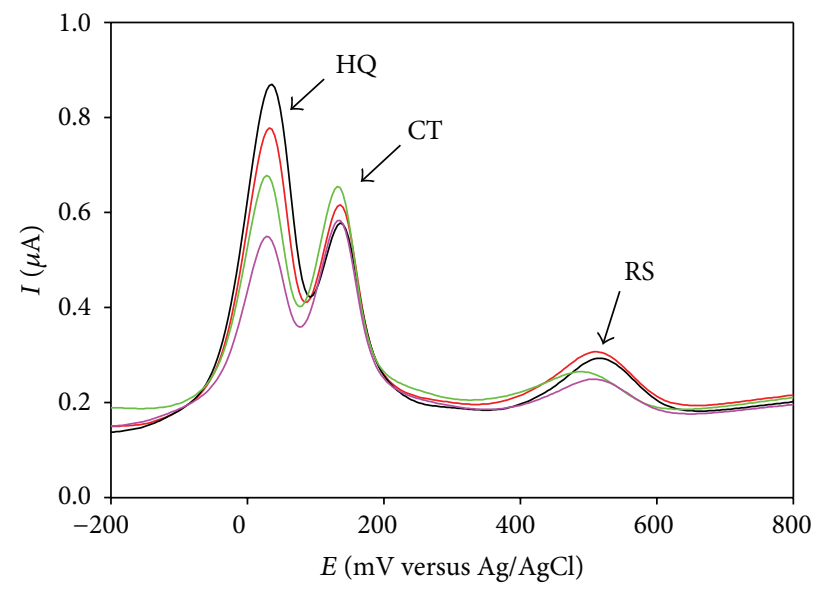

(a)

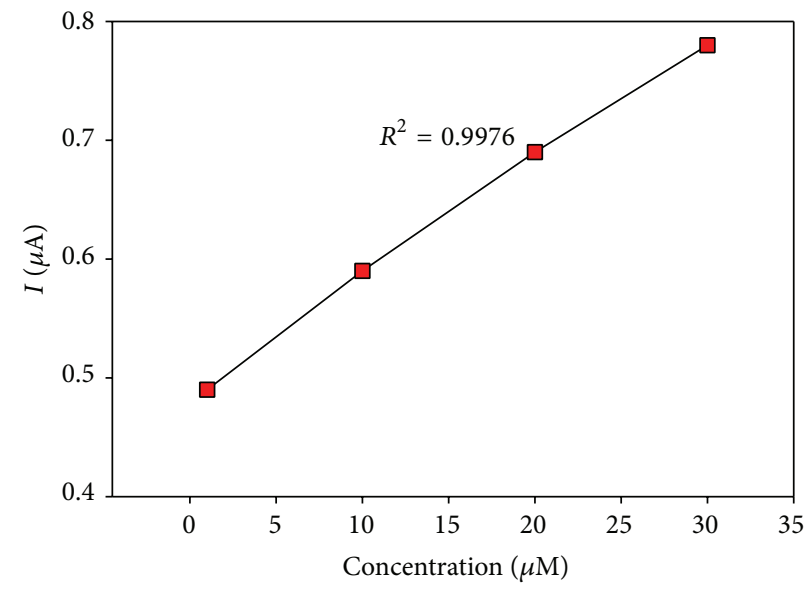

(b)

FIGURE 8: (a) Differential pulse voltammogram (DPV) HQ in the presence of CT and RS PGA modified GCE in PBS. (b) Calibration curve for the quantitative determination of HQ in ternary mixture with CT and RS. 
and quantify dihydroxybenzenes as pollutants and contaminants in real environmental samples. Though only qualitative studies could be done at this point, further studies towards the improvement of detection and quantitative estimation is required and will be reported in due course.

\section{Conflict of Interests}

The authors declare that there is no conflict of interests regarding the publication of this paper.

\section{References}

[1] J. Wang, J.-N. Park, X.-Y. Wei, and C. W. Lee, "Roomtemperature heterogeneous hydroxylation of phenol with hydrogen peroxide over $\mathrm{Fe}^{2+}, \mathrm{Co}^{2+}$ ion-exchanged $\mathrm{Na} \beta$ zeolite," Chemical Communications, no. 5, pp. 628-629, 2003.

[2] A. J. S. Ahammad, M. M. Rahman, G.-R. Xu, S. Kim, and J.J. Lee, "Highly sensitive and simultaneous determination of hydroquinone and catechol at poly(thionine) modified glassy carbon electrode," Electrochimica Acta, vol. 56, no. 14, pp. 52665271, 2011.

[3] T. Xie, Q. Liu, Y. Shi, and Q. Liu, "Simultaneous determination of positional isomers of benzenediols by capillary zone electrophoresis with square wave amperometric detection," Journal of Chromatography A, vol. 1109, no. 2, pp. 317-321, 2006.

[4] A. J. S. Ahammad, S. Sarker, M. A. Rahman, and J.-J. Lee, "Simultaneous determination of hydroquinone and catechol at an activated glassy carbon electrode," Electroanalysis, vol. 22, no. 6, pp. 694-700, 2010.

[5] H. Cui, C. He, and G. Zhao, "Determination of polyphenols by high-performance liquid chromatography with inhibited chemiluminescence detection," Journal of Chromatography A, vol. 855, no. 1, pp. 171-179, 1999.

[6] Y. Ding, W. Liu, Q. Wu, and X. Wang, "Direct simultaneous determination of dihydroxybenzene isomers at C-nanotubemodified electrodes by derivative voltammetry," Journal of Electroanalytical Chemistry, vol. 575, no. 2, pp. 275-280, 2005.

[7] L. Wang, Y. Zhang, Y. Du, D. Lu, Y. Zhang, and C. Wang, "Simultaneous determination of catechol and hydroquinone based on poly (diallyldimethylammonium chloride) functionalized graphene-modified glassy carbon electrode," Journal of Solid State Electrochemistry, vol. 16, no. 4, pp. 1323-1331, 2012.

[8] G. Marrubini, E. Calleri, T. Coccini, A. F. Castoldi, and L. Manzo, "Direct analysis of phenol, catechol and hydroquinone in human urine by coupled-column HPLC with fluorimetric detection," Chromatographia, vol. 62, no. 1-2, pp. 25-31, 2005.

[9] M. F. Pistonesi, M. S. Di Nezio, M. E. Centurión, M. E. Palomeque, A. G. Lista, and B. S. Fernández Band, "Determination of phenol, resorcinol and hydroquinone in air samples by synchronous fluorescence using partial least-squares (PLS)," Talanta, vol. 69, no. 5, pp. 1265-1268, 2006.

[10] H. Cui, Q. Zhang, A. Myint, X. Ge, and L. Liu, "Chemiluminescence of cerium(IV)-rhodamine 6G-phenolic compound system," Journal of Photochemistry and Photobiology A, vol. 181, no. 2-3, pp. 238-245, 2006.

[11] P. Nagaraja, R. A. Vasantha, and K. R. Sunitha, "A sensitive and selective spectrophotometric estimation of catechol derivatives in pharmaceutical preparations," Talanta, vol. 55, no. 6, pp. 1039-1046, 2001.
[12] S. C. Moldoveanu and M. Kiser, "Gas chromatography/mass spectrometry versus liquid chromatography/fluorescence detection in the analysis of phenols in mainstream cigarette smoke," Journal of Chromatography A, vol. 1141, no. 1, pp. 90-97, 2007.

[13] N. Guan, Z. Zeng, Y. Wang, E. Fu, and J. Cheng, "Open tubular capillary electrochromatography in fused-silica capillaries chemically bonded with macrocyclic dioxopolyamine," Analytica Chimica Acta, vol. 418, no. 2, pp. 145-151, 2000.

[14] A. J. S. Ahammad, N. C. D. Nath, G.-R. Xu, S. Kim, and J.J. Lee, "Interference-free determination of dopamine at the poly(thionine)-modified glassy carbon electrode," Journal of the Electrochemical Society, vol. 158, no. 6, pp. F106-F110, 2011.

[15] M. A. Ghanem, "Electrocatalytic activity and simultaneous determination of catechol and hydroquinone at mesoporous platinum electrode," Electrochemistry Communications, vol. 9, no. 10, pp. 2501-2506, 2007.

[16] M. Li, F. Ni, Y. Wang et al., "Sensitive and facile determination of catechol and hydroquinone simultaneously under coexistence of resorcinol with a $\mathrm{Zn} / \mathrm{Al}$ layered double hydroxide film modified glassy carbon electrode," Electroanalysis, vol. 21, no. 13, pp. 1521-1526, 2009.

[17] J. Peng and Z. Gao, "Influence of micelles on the electrochemical behaviors of catechol and hydroquinone and their simultaneous determination," Analytical and Bioanalytical Chemistry, vol. 384, no. 7-8, pp. 1525-1532, 2006.

[18] Y. Zhang and J. B. Zheng, "Comparative investigation on electrochemical behavior of hydroquinone at carbon ionic liquid electrode, ionic liquid modified carbon paste electrode and carbon paste electrode," Electrochimica Acta, vol. 52, no. 25, pp. 7210-7216, 2007.

[19] H. Qi and C. Zhang, "Simultaneous determination of hydroquinone and catechol at a glassy carbon electrode modified with multiwall carbon nanotubes," Electroanalysis, vol. 17, no. 10, pp. 832-838, 2005

[20] L. Wang, P. Huang, J. Bai, H. Wang, L. Zhang, and Y. Zhao, "Covalent modification of a glassy carbon electrode with penicillamine for simultaneous determination of hydroquinone and catechol," Microchimica Acta, vol. 158, no. 1, pp. 151-157, 2007.

[21] L. Wang, P. Huang, J. Bai, H. Wang, L. Zhang, and Y. Zhao, "Simultaneous electrochemical determination of phenol isomers in binary mixtures at a poly (phenylalanine) modified glassy carbon electrode," International Journal of Electrochemical Science, vol. 1, no. 8, pp. 403-413, 2006.

[22] L. Wang, P. Huang, H. Wang, J. Bai, L. Zhang, and Y. Zhao, "Covalent modification of glassy carbon electrode with aspartic acid for simultaneous determination of hydroquinone and catechol," Annali di Chimica, vol. 97, no. 5-6, pp. 395-404, 2007.

[23] L. Wang, P. Huang, H. Wang, J. Bai, L. Zhang, and Y. Zhao, "Electrocatalytic response of hydroquinone and catechol at polyglycine modified glassy carbon electrode," International Journal of Electrochemical Science, vol. 2, pp. 216-225, 2007.

[24] A. A. Shaikh, S. K. Saha, P. K. Bakshi, A. Hossain, and A. J. Saleh Ahmed, "Poly(brilliant cresyl blue)-modified electrode for highly sensitive and simultaneous determination of hydroquinone and catechol," Journal of the Electrochemical Society, vol. 160, no. 4, pp. B37-B42, 2013.

[25] L. Ma and G.-C. Zhao, "Simultaneous determination of hydroquinone, catechol and resorcinol at graphene doped carbon ionic liquid electrode," International Journal of Electrochemistry, vol. 2012, Article ID 243031, 8 pages, 2012. 
[26] L. A. Alshahrani, X. Li, H. Luo et al., "The simultaneous electrochemical detection of catechol and hydroquinone with $\left[\mathrm{Cu}(\mathrm{Sal}-\beta\right.$-Ala $\left.)(3,5-\mathrm{DMPz})_{2}\right] /$ SWCNTs/GCE," Sensors, vol. 14, no. 12, pp. 22274-22284, 2014.

[27] T. C. Canevari, L. T. Arenas, R. Landers, R. Custodio, and Y. Gushikem, "Simultaneous electroanalytical determination of hydroquinone and catechol in the presence of resorcinol at an $\mathrm{SiO}_{2} / \mathrm{C}$ electrode spin-coated with a thin film of $\mathrm{Nb}_{2} \mathrm{O}_{5}$," Analyst, vol. 138, no. 1, pp. 315-324, 2013.

[28] W. Liu, L. Wu, X. Zhang, and J. Chen, "Simultaneous electrochemical determination of hydroquinone, catechol and resorcinol at nitrogen doped porous carbon nanopolyhedronsmultiwall carbon nanotubes hybrid materials modified glassy carbon electrode," Bulletin of the Korean Chemical Society, vol. 35, no. 1, pp. 204-210, 2014.

[29] Z. Wang, S. Li, and Q. Lv, "Simultaneous determination of dihydroxybenzene isomers at single-wall carbon nanotube electrode," Sensors and Actuators B, vol. 127, no. 2, pp. 420-425, 2007.

[30] D. Zhang, Y. Peng, H. Qi, Q. Gao, and C. Zhang, "Application of multielectrode array modified with carbon nanotubes to simultaneous amperometric determination of dihydroxybenzene isomers," Sensors and Actuators, B: Chemical, vol. 136, no. 1, pp. 113-121, 2009. 

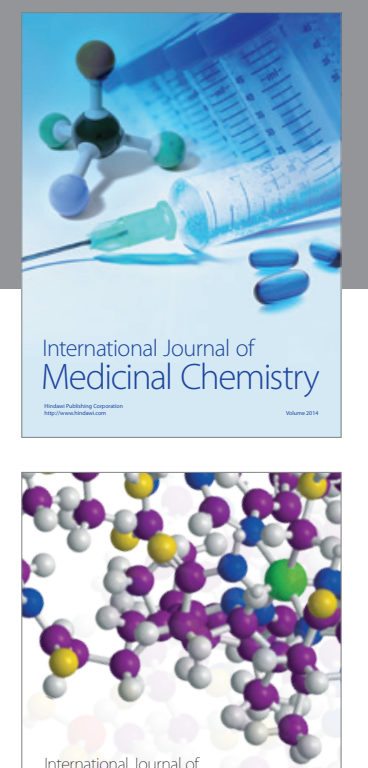

\section{Carbohydrate} Chemistry

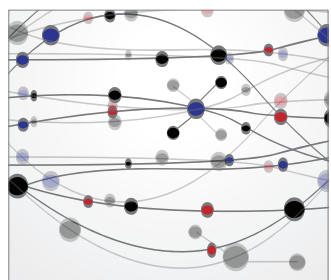

The Scientific World Journal
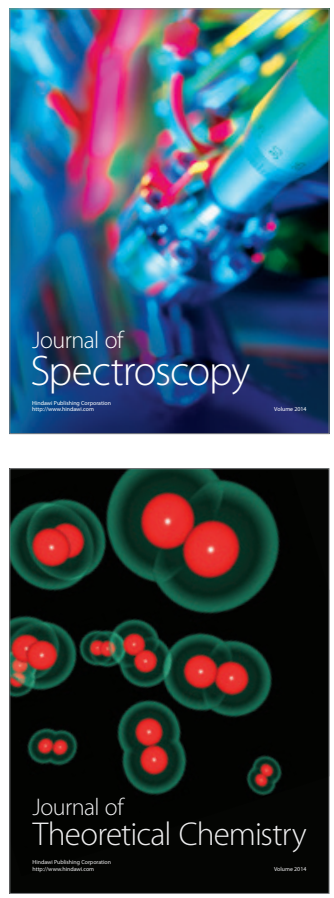
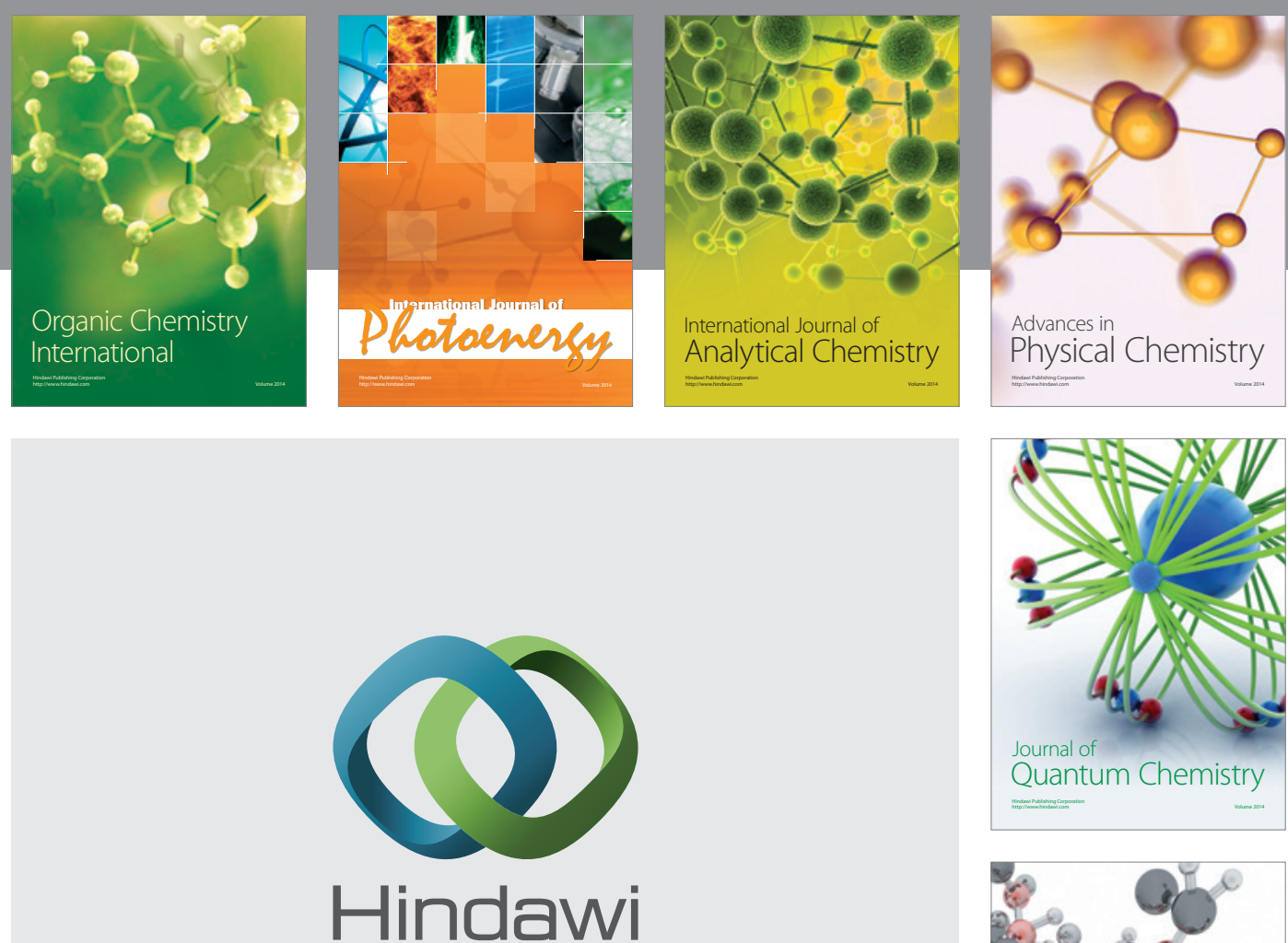

Submit your manuscripts at

http://www.hindawi.com

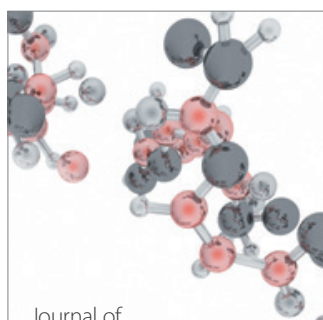

Analytical Methods

in Chemistry

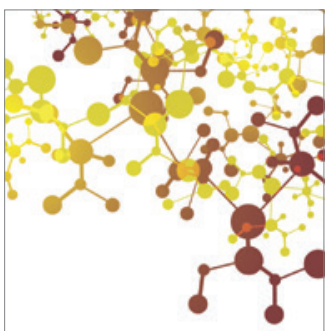

Journal of

Applied Chemistry

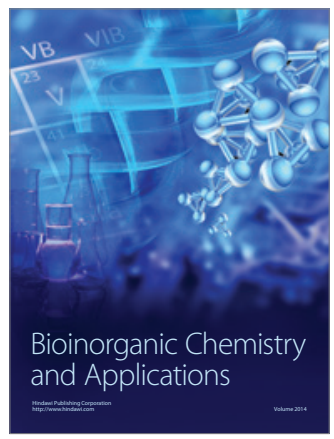

Inorganic Chemistry
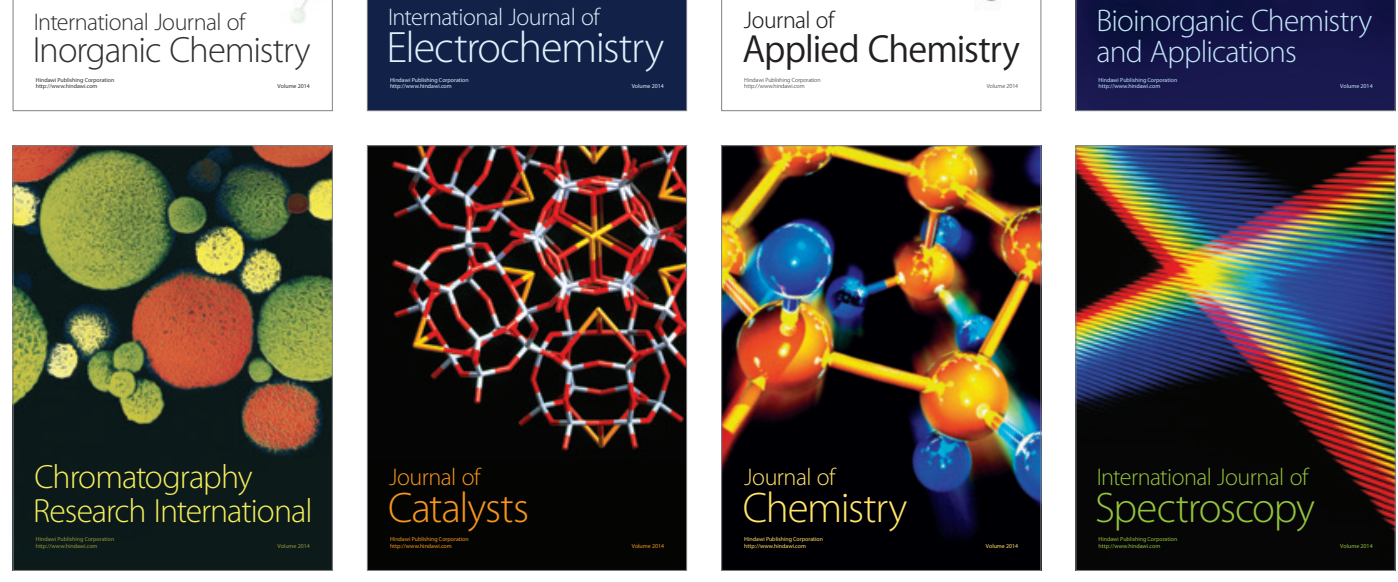